\title{
A Rank-Deficient and Sparse Penalized Optimization Model for Compressive Indoor Radar Target Localization
}

\author{
Van Ha Tang, Van-Giang Nguyen \\ Faculty of Information Technology, Le Quy Don Technical University, Hanoi, Vietnam \\ Correspondence: Van Ha Tang, hatv@lqdtu.edu.vn \\ Communication: received 14 May 2019, revised 2 September 2019, accepted 4 October 2019 \\ Online publication: 13 February 2020, Digital Object Identifier: 10.21553/rev-jec.236 \\ The associate editor coordinating the review of this article and recommending it for publication was Prof. Nguyen Linh Trung.
}

\begin{abstract}
We introduce a low-rank and sparse penalized optimization model for solving the problem of radar imaging of indoor targets in the presence of strong wall clutter from compressed data measurements. Compressive through-wall radar imaging (TWRI) accelerates data collection and reduces operation cost, but incomplete radar data makes wall clutter mitigation and target image reconstruction become more challenging. This paper aims to tackle these difficulties by formulating the task of wall clutter suppression and target image formation as a penalized minimization problem with low-rank and sparse regularizers. The former penalty is used to model the low-dimensional attribute of the wall reflections and the later regularizer is used to represent the image of the behind-the-wall targets. We develop an iterative algorithm based on the forward-backward proximal gradient technique to solve the regularized minimization problem, which removes wall interferences and forms an indoor target image simultaneously. The effectiveness of the proposed approach is validated using extensive experiments on both simulated and real radar data.
\end{abstract}

Keywords- Through-wall radar imaging, wall clutter mitigation, compressed sensing, target image reconstruction, proximal gradient techniques.

\section{INTRODUCTION}

Through-wall radar (TWR) imaging is an emerging and powerful technology for sensing targets behind walls and other opaque structures. The ability of penetrating through-wall is very useful for numerous potential applications in military operations, civilian applications, and search-and-secure missions [1-3]. In such applications, it is highly demanding for the development of a successful TWRI system that can provide highquality images of desired targets and combat unwanted interferences of wall clutter. The imaging system also provides high-resolution images with fast data collection and optimal data storage. To this end, this article introduces an efficient approach that performs wall clutter mitigation and target image formation in compressive sensing operations.

Conventionally, TWRI techniques require a complete dataset to generate an image of indoor targets using backprojection, such as delay-and-sum beamforming [1, $2,4]$. In other words, such techniques are effective for image formation only for the case in which all the antennas and frequencies are available for data acquisition. However, this data collection mode makes data acquisition prolonged and system storage ineffective. To accelerate data collection and provide high-resolution imaging, several TWRI approaches have been considered using the compressive sensing (CS) framework [57]. As CS is a powerful signal processing technique that allows compressive sampling and precise reconstruction of sparse signals, it has been applied to TWRI for image formation from far reduced measurements [8-
10]. Using CS, the task of image formation is formulated as an $\ell_{1}$ penalized minimization problem, in which the $\ell_{1}$ regularizer is used to promote the sparseness of the target scene. It has been shown that this minimization model is suitable for the situations where strong wall clutter has been completely removed prior to image reconstruction through background subtraction. Having the access to a background scene, however, is impossible in many practice operations. In fact, the presence of wall clutter causes the $\ell_{1}$-penalized approaches ineffective; they reconstruct only the pixels belonging to wall clutter that tend to dominate the target pixels, making target detection very difficult.

To alleviate wall interferences, the problem of target image formation in conjunction with wall clutter mitigation has been considered in several CS-based studies that consist of two major stages [11-14]. The first stage performs wall clutter mitigation, followed by image formation in the second stage. In the wall clutter suppression stage, a full data volume needs to be estimated from the reduced dataset before spatial filtering [15] or subspace projection [16] techniques are applied to the estimated data for wall clutter removal. The wall-clutter free data are then used in the second stage for image formation through an $\ell_{1}$ minimization. Due to the multistage signal processing, these CSbased approaches may be affected by suboptimality and uncertainly; the performances of wall clutter mitigation and target image formation are sensitive to the estimation error arising in the signal recovery stage.

Instead of performing multistage independently, the key idea of the proposed approach in this paper is 
to perform wall clutter mitigation and target image reconstruction in CS TWRI simultaneously through an optimization model. This optimization model is formulated by incorporating two intrinsic signal structures: (1) low-dimensional structure of wall clutter and (2) the sparsity profile of the target scene. The former structure is due to the fact that the electromagnetic reflections from the front wall received along the antenna array are highly correlated. As a result, if the wall antenna signals are arranged as columns of a matrix, this matrix is lowrank. The later attribute of the model is because target pixels occupy only a small region in the form image. In other words, the target image is sparse. Intuitively, we could perform these two important tasks even better if we represent the model more precisely and completely. By incorporating further prior knowledge into the model, we hope to improve the model performance.

The idea of joint wall clutter mitigation and image formation, and preliminary results have been presented in [17]. This paper extends this work in three respects: model formulation, iterative algorithm, and experimental evaluation. The problem formulation is described completely in this paper, for both full and compressive sensing operations. Furthermore, the problem formulation is discussed and compared with the two existing techniques of DS beamforming and multistage CS-based models, which highlights the advantages of the proposed model. In terms of algorithm design, this paper presents rigorous steps for solving the joint nuclear-norm and $\ell_{1}$-norm regularized least squares (LS) minimization problem, based on the proximal forward-backward splitting framework [18-20]. This generic technique, its application to TWRI, and how the proximal evaluations of the two key operators, namely singular value thresholding and soft-thresholding to overcome the challenging nonsmooth nature of the penalty terms are detailed in this paper. Algorithm analysis, its convergence, and computational complexity are discussed. Extensive simulations and experiments are conducted to evaluate the performance of the proposed model. In addition, performance comparisons with several state-of-the-art methods are described and analyzed in different CS settings.

This article is organized as follows. Section 2 presents TWR signal model briefly. Section 3 describes the problem formulation of the proposed low-rank and sparse regularized LS model and presents an iterative algorithm based on the forward-backward proximal technique for wall clutter suppression and indoor target image formation. Experimental evaluation on simulated and real radar data is given in Section 4 and finally, Section 5 concludes the paper.

\section{Through-Wall Radar Signal Model}

This section gives a brief introduction about the signal model of a monostatic stepped-frequency synthetic aperture radar system used to sense targets residing behind the wall. Such targets are imaged by placing a transceiver in front of the wall at a standoff distance $z_{\text {off }}$. This sensor transceives the signal and then moves to another places along a horizontal line parallel to the wall to interrogate the scene. Suppose an $M$ steppedfrequency signal has been used to image $P$ indoor targets by a synthesized $N$ antennas. Let $z_{m, n}$ denote the received signal for the $m$ th frequency by the $n$th antenna. This signal is modeled as a superposition of the wall clutter $z_{m, n}^{w}$, target signal $z_{m, n}^{t}$, and noise $v_{m, n}$ :

$$
z_{m, n}=z_{m, n}^{w}+z_{m, n}^{t}+v_{m, n} .
$$

The wall component $z_{m, n}^{w}$ is modeled as the sum of wall reverberations $[11,21]$

$$
z_{m, n}^{w}=\sum_{r=1}^{R} \sigma_{w} a_{r} e^{-j 2 \pi f_{m} \tau_{n, w}^{r}} .
$$

Here, $\sigma_{w}$ denotes the wall reflectivity, $R$ is the number of wall reverberations, $a_{r}$ is the path loss of the $r$ th wall reverberation, and $\tau_{n, w}^{r}$ represents the $r$ th wall return travel delay. This round propagation delay is similar along the antenna array and given by $\tau_{n, w}^{r}=\frac{2 z_{\text {off }}}{c}$ with $c$ being the speed of light in free-space. The target signal can be modeled as a superposition of all the targets present in the imaged scene $[12,13]$

$$
z_{m, n}^{t}=\sum_{p=1}^{P} \sigma_{p} e^{-j 2 \pi f_{m} \tau_{n, p}}
$$

where $\sigma_{p}$ is the $p$ th target reflectivity, and $\tau_{n, p}$ denotes the round-trip signal travel time from the $n$th antenna to the $p$ th target. Let $\mathbf{z}_{n}=\left[z_{1, n}, \ldots, z_{M, n}\right]^{T}$ denote the column vector formulated by stacking $M$ frequency measurements collected along the $n$th antenna. The signal model in (1) can be represented in vector-form:

$$
\mathbf{z}_{n}=\mathbf{z}_{n}^{w}+\mathbf{z}_{n}^{t}+\boldsymbol{v}_{n} .
$$

Arranging the $N$ vectors $\mathbf{z}_{n}$, for $n=1, \ldots, N$, as columns of the matrix $\mathbf{Z} \in \mathbb{C}^{M \times N}$, we have the following matrix form:

$$
\mathbf{Z}=\left[\mathbf{z}_{1}, \ldots, \mathbf{z}_{N}\right]=\mathbf{Z}^{w}+\mathbf{Z}^{t}+\mathbf{Y} .
$$

For target image formation, the target space considered as a rectangular grid is partitioned into $Q$ pixels along the crossrange (horizontal) and downrange (vertical) directions. Let $\mathbf{s}$ be a vector that represents the target grid. This grid relates to the reflectivity of the targes as follows:

$$
s_{q}= \begin{cases}\sigma_{p}, & \text { if the } p \text { th target occupies the } q \text { th pixel; } \\ 0, & \text { otherwise. }\end{cases}
$$

This implies that the value $s_{q}$ of the $q$ th pixel on the grid is modeled as a weighted indicator function used to represent the $p$ th target reflectivity. In TWRI, the targets tend to occupy as groups consisting of points populated accurately on the image-pixel locations. From (3) and (4), we can relate the target measurement vector $\mathbf{z}_{n}^{t}$ to the image scene $\mathbf{s}=\left[s_{1}, \ldots, s_{Q}\right]^{T}$, through a dictionary matrix $\Psi_{n} \in \mathbb{C}^{M \times Q}$ :

$$
\mathbf{z}_{n}^{t}=\mathbf{\Psi}_{n} \mathbf{s} .
$$

Here, the $(m, q)$ th element of matrix $\Psi_{n}$ is computed 
as $\psi_{n}(m, q)=\exp \left(-j 2 \pi f_{m} \tau_{n, q}\right)$. In this computation, the term $\tau_{n, q}$ plays an important role in focusing the targets because it takes into accout the signal delays before, through, and after the wall in its calculation $[2,4,22]$. Arranging all target measurement vectors, $\mathbf{z}^{t}=\left[\left(\mathbf{z}_{1}^{t}\right)^{T}, \ldots,\left(\mathbf{z}_{N}^{t}\right)^{T}\right]^{T}$, and dictionary matrices, $\Psi=\left[\Psi_{1}^{T}, \ldots, \Psi_{N}^{T}\right]^{T}$, for $N$ antennas, we have the linear model,

$$
\mathbf{z}^{t}=\operatorname{vec}\left(\mathbf{Z}^{t}\right)=\mathbf{\Psi} \mathbf{s},
$$

hereafter $\operatorname{vec}(\cdot)$ denotes the vectorization operator forming a composite column vector by stacking the columns of a matrix in lexicographic order.

It is followed from (8) that the target image $\mathbf{s}$ can be estimated from the target signal $\mathbf{z}^{t}$ using DS beamforming or direct CS technique. The DS beamforming generates the image $\mathbf{s}$ by premultiplying the target signal $\mathbf{z}^{t}$ with the adjoint operator $\mathbf{\Psi}^{H}$ :

$$
\mathbf{s}=\mathbf{\Psi}^{H} \mathbf{z}^{t} .
$$

While the DS beamforming does not exploit any prior knowledge, the direct CS guarantees the sparsity of $\mathbf{s}$ and yields a target image by solving an $\ell_{1}$ regularization problem:

$$
\mathbf{s}=\arg \min _{\mathbf{s}}\left\{\frac{1}{2}\left\|\mathbf{z}^{t}-\mathbf{\Psi} \mathbf{s}\right\|_{2}^{2}+\lambda\|\mathbf{s}\|_{1}\right\},
$$

with $\lambda$ being a positive parameter. It is worth noting here that the target signal $\mathbf{z}^{t}$ is not available for image formation; in fact, we have only the the radar signal $\mathbf{z}$ that comprises the dominated wall clutter $\mathbf{z}^{w}$, target signal $\mathbf{z}^{t}$, plus noise, see (4). Due to the dominance of the wall component $\mathbf{z}^{w}$, in the formed target image $\mathbf{s}$ by either DS beamforming in (9) or direct CS technique in (10), the wall clutter pixels mask those of the targets, making target detection very difficult. Therefore, prior to target image formation, strong wall clutter needs to be suppressed. Unfortunately, performing wall clutter mitigation is even more challenging in CS operations where the radar data is incomplete. Wall clutter mitigation techniques, such as subspace projection [16], can be applied if the same frequency measurements are available at all antennas. In general compressed sensing TWR, however, only a subset of frequency samples is acquired, which may vary from one spatial position to another. To address this issue, multistage CS-based imaging techniques have been considered, where the missing measurements are first estimated, followed by wall clutter mitigation applied to full recovered measurements, and target image formation. To alleviate the shortcomings of multistage processing, this paper proposes a model that comprises rank-deficient and sparsity regularizations to mitigate wall clutter and reconstruct a target image jointly.

\section{Rank-Deficient and Sparse Regularized TWRI}

In this section, we first describe the formulation of the rank-deficient and sparse regularized optimization problem arising in TWRI in Subsection 3.1. Then, Subsection 3.2 presents a proximal gradient-based iterative algorithm to solve the nuclear and $\ell_{1}$-norm optimization problem, capturing wall clutter and yielding an indoor target image.

\subsection{Nuclear and $\ell_{1}$-norm Regularized LS Problem}

The signal model presented in Equations (4) and (5) assumes a full set of $(M \times N)$ measurements collected at all $N$ antennas using $M$ frequencies. For fast data acquisition and efficient data storage, we consider the problem in the CS operation where only a reduced set of measurements is collected for imaging targets. Suppose that a subset of $K$ data samples $(K \ll M \times N)$ is acquired. The reduced dataset can be obtained by using a selection matrix $\boldsymbol{\Phi} \in \mathbb{R}^{K \times M N} ; \boldsymbol{\Phi}$ has only one nonzero element (equal to 1 ) in each row that represents the selected frequency for a particular antenna used. The compressive measurement vector $\mathbf{y} \in \mathbb{C}^{K}$ is related to the full measurement data $\mathbf{Z}$ as,

$$
\mathbf{y}=\mathbf{\Phi} \operatorname{vec}(\mathbf{Z})=\mathcal{A}(\mathbf{Z}) \text {. }
$$

In (11), $\mathcal{A}$ can be regarded as a linear operator acting on the space of $M \times N$ matrices, i.e., $\mathcal{A}: \mathbb{C}^{M \times N} \rightarrow \mathbb{C}^{K}$. Note here that the matrix $\mathbf{Z}$ can be obtained from $\mathbf{y}$ via the adjoint operator $\mathcal{A}^{*}$ as $\mathbf{Z}=\operatorname{mat}\left(\boldsymbol{\Phi}^{\dagger} \mathbf{y}\right)=\mathcal{A}^{*}(\mathbf{y})$, where ${ }^{+}$is the pseudo-inverse operator and mat is the operator converting a column vector having $M N$ entries into an $M \times N$ matrix. Combining (5) and (11) yields

$$
\mathbf{y}=\boldsymbol{\Phi} \operatorname{vec}(\mathbf{Z})=\boldsymbol{\Phi} \operatorname{vec}\left(\mathbf{Z}^{w}\right)+\boldsymbol{\Phi} \operatorname{vec}\left(\mathbf{Z}^{t}\right)+\boldsymbol{\Phi} \operatorname{vec}(\mathbf{Y}) \text {. }
$$

Exploiting the model that relates the target signal and the image in (8), $\operatorname{vec}\left(\mathbf{Z}^{t}\right)=\mathbf{z}^{t}=\boldsymbol{\Psi} \mathbf{s}$, we have the linear model

$$
\mathbf{y}=\boldsymbol{\Phi} \operatorname{vec}\left(\mathbf{Z}^{w}\right)+\boldsymbol{\Phi} \Psi \mathbf{s}+\boldsymbol{\Phi} \operatorname{vec}(\mathbf{Y}) .
$$

Given the measurement vector $\mathbf{y}$, the aim is to estimate a matrix $\mathbf{Z}^{w}$ carrying wall clutter and a vector $\mathbf{s}$ representing the target image. Towards this aim, prior knowledge about the model needs to be considered. In other words, the attributes of $\mathbf{Z}^{w}$ and $\mathbf{s}$ should be exploited for the constraints of the solutions. Here, two important structures are used. The first structure is the rank-deficient of $\mathbf{Z}^{w}$, which reflects the fact that its columns are highly correlated. The rank-deficient of $\mathbf{Z}^{w}$ is also evident from the model in Equation (2) that the wall reflection is similar among the antenna location. The second assumption is the sparseness of the target image s, which holds true in practice as target pixels occupy only a small part of the whole imaged scene. It is worth noting that the low-rank and sparse constraints can be modeled efficiently through their convex relaxations: low-rank via nuclear norm $[23,24]$ and sparse via $\ell_{1}$-norm [6, 7]. By so doing, we can attain the wall component $\mathbf{Z}^{w}$ and the image $\mathbf{s}$ as the solution to the following optimization problem:

$$
\begin{array}{ll}
\min _{\mathbf{Z}^{w}, \mathbf{s}} & \left\|\mathbf{Z}^{w}\right\|_{*}+\lambda\|\mathbf{s}\|_{1} \\
\text { subject to } & \left\|\mathbf{y}-\left[\mathcal{A}\left(\mathbf{Z}^{w}\right)+\mathbf{D} \mathbf{s}\right]\right\|_{2}^{2} \leq \epsilon,
\end{array}
$$


where we have defined $\mathbf{D}=\boldsymbol{\Phi} \boldsymbol{\Psi},\left\|\mathbf{Z}^{w}\right\|_{*}$ is the nuclearnorm defined as the sum of the singular values of $\mathbf{Z}^{w},\left\|\mathbf{Z}^{w}\right\|_{*}=\sum_{j=1}^{J} \lambda_{j}\left(\mathbf{Z}^{w}\right)$ with $\lambda_{j}\left(\mathbf{Z}^{w}\right)$ being the $j$ th largest singular value of matrix $\mathbf{Z}^{w}$ of rank at most $J$, $\|\mathbf{s}\|_{1}$ is the $\ell_{1}$-norm defined as the sum of absolute entries of $\mathbf{s},\|\mathbf{s}\|_{1}=\sum_{q=1}^{Q}\left|s_{q}\right|, \lambda$ is a regularization parameter, and $\epsilon$ is a noise bound. Problem (14) can be handled efficiently by casting into the standard Lagrangian form:

$$
\begin{aligned}
\min _{\mathbf{Z}^{w}, \mathbf{s}}\{ & f\left(\mathbf{Z}^{w}, \mathbf{s}\right) \equiv \frac{1}{2}\left\|\mathbf{y}-\left[\mathcal{A}\left(\mathbf{Z}^{w}\right)+\mathbf{D} \mathbf{s}\right]\right\|_{2}^{2} \\
& \left.+\gamma\left\|\mathbf{Z}^{w}\right\|_{*}+\lambda\|\mathbf{s}\|_{1}\right\} .
\end{aligned}
$$

Convex theory has proved that the solutions to (14) and (15) are equivalent if $\gamma$ and $\epsilon$ obey certain relationships [25]. Minimizing $f\left(\mathbf{Z}^{w}, \mathbf{s}\right)$ produces the wall clutter matrix $\mathbf{Z}^{w}$ and indoor target image $\mathbf{s}$ jointly. Before presenting the algorithm to minimize $f\left(\mathbf{Z}^{w}, \mathbf{s}\right)$, it is worth noting here that in comparison with the conventional DS beamforming model in (9) considering no prior knowledge, and the direct CS-based technique in (10) taking into account only the sparsity of target image, the proposed approach in (15) encompasses a more complete and precise knowledge, which can enhance model performance.

\subsection{Iterative Algorithm}

This subsection introduces an iterative algorithm to solve the low-rank and sparse regularized LS problem in (15). Although this minimization problem is convex, it is complicated to solve it directly due to the nonsmoothness of the regularization terms. To handle this issue, we develop an algorithm based on the proximal forward-backward splitting (PFBS) technique. Before presenting the algorithm, let us consider a generic case of minimizing a composite objective function:

$$
\min _{\mathbf{X}}\{f(\mathbf{X})=g(\mathbf{X})+h(\mathbf{X})\},
$$

where $g(\mathbf{X})$ is convex and differentiable with a $C$ Lipschitz continuous gradient $\nabla g$, and $h(\mathbf{X})$ is convex but not necessary smooth. PFBS handles Problem (16) by an iterative scheme that involves a forward gradient evaluation of $g(\mathbf{X})$ and a backward proximal operator of $h(\mathbf{X})$. Let $\mathbf{X}_{t}$ denote an estimate of the solution at the $t$ th iteration. The next estimate is obtained by

$$
\mathbf{X}_{t+1}=\underbrace{\operatorname{prox}_{\mu_{t} h}}_{\text {backward step }} \underbrace{\left(\mathbf{X}_{t}-\mu_{t} \nabla g\left(\mathbf{X}_{t}\right)\right)}_{\text {forward step }} .
$$

Here, the stepsize $\mu_{t}$ satisfies $0<\mu_{t} \leq 1 / C$ to ensure convergence, and the proximal operator is defined as [20]

$$
\operatorname{prox}_{\mu_{t} h}(\mathbf{Z})=\arg \min _{\mathbf{X}}\left\{\frac{1}{2}\|\mathbf{Z}-\mathbf{X}\|_{F}^{2}+\mu_{t} h(\mathbf{X})\right\} .
$$

In other words, let $\mathbf{Z}_{t}$ denote the result of the gradient step evaluated using the current estimate $\mathbf{X}_{t}$,

$$
\mathbf{Z}_{t}=\mathbf{X}_{t}-\mu_{t} \nabla g\left(\mathbf{X}_{t}\right)
$$

The next estimate of the solution is obtained by proximal evaluation,

$$
\mathbf{X}_{t+1}=\operatorname{prox}_{\mu_{t} h}\left(\mathbf{Z}_{t}\right) .
$$

As the forward gradient is simple, the proximal evaluation is the main computation cost. Hence, this algorithm is computational-efficient if the proximal operator has a closed-form solution.

The generic PFBS scheme in (19)-(20) is used to minimize Problem (15). Let $\left(\mathbf{Z}_{t}^{w}, \mathbf{s}_{t}\right)$ denote an estimate of the wall component and target image at the $t$ th iteration. The solution is obtained by the following forward gradient and backward proximal evaluations:

$$
\begin{aligned}
\mathbf{Z}_{t}=\mathbf{Z}_{t}^{w}+\mathcal{A}^{*}\left(\mathbf{D} \mathbf{s}_{t}\right)-\mathcal{A}^{*}\left(\mathcal{A}\left(\mathbf{Z}_{t}^{w}\right)+\mathbf{D} \mathbf{s}_{t}-\mathbf{y}\right), \\
\left(\mathbf{Z}_{t+1}^{w}, \mathbf{s}_{t+1}\right)=\arg \min _{\mathbf{Z}^{w}, \mathbf{s}}\left\{\frac{1}{2}\left\|\mathbf{Z}_{t}-\mathbf{Z}^{w}-\mathcal{A}^{*}(\mathbf{D} \mathbf{s})\right\|_{F}^{2}\right. \\
\left.+\gamma\left\|\mathbf{Z}^{w}\right\|_{*}+\lambda\|\mathbf{s}\|_{1}\right\} .
\end{aligned}
$$

In Equations (21) and (22), the stepsize $\mu_{t}$ is omitted because it is set to $\mu_{t}=1 / C$ with $C=\lambda_{\max }\left(\boldsymbol{\Phi}^{T} \boldsymbol{\Phi}\right)=1$. Since the two variables $\mathbf{Z}^{w}$ and $\mathbf{s}$ are separable, Problem (22) can be handled using the variable splitting technique, leading to solving the following two subproblems:

$$
\begin{gathered}
\mathbf{Z}_{t+1}^{w}=\arg \min _{\mathbf{Z}^{w}}\left\{\frac{1}{2}\left\|\left[\mathbf{Z}_{t}-\mathcal{A}^{*}\left(\mathbf{D} \mathbf{s}_{t}\right)\right]-\mathbf{Z}^{w}\right\|_{F}^{2}\right. \\
\left.+\gamma\left\|\mathbf{Z}^{w}\right\|_{*}\right\}, \\
\mathbf{s}_{t+1}=\arg \min _{\mathbf{s}}\left\{\frac{1}{2}\left\|\mathbf{Z}_{t}-\mathbf{Z}_{t+1}^{w}-\mathcal{A}^{*}(\mathbf{D} \mathbf{s})\right\|_{F}^{2}+\lambda\|\mathbf{s}\|_{1}\right\} .
\end{gathered}
$$

The remaining task is to solve Subproblems (23) and (24), which can be handled efficiently via shrinkage/soft-thresholding techniques. In particular, the LS problem regularized by the nuclear-norm term in (23) is solved using the singular value soft-thresholding (SVT) technique $[24,26]$. The solution is obtained by applying an SVT operator, $\mathcal{S}_{\gamma}(\cdot)$, to the input matrix:

$$
\mathbf{Z}_{t+1}^{w}=\mathcal{S}_{\gamma}\left(\mathbf{Z}_{t}-\mathcal{A}^{*}\left(\mathbf{D} \mathbf{s}_{t}\right)\right) \text {. }
$$

In general, the SVT operator $\mathcal{S}_{\tau}(\mathbf{Z})$ comprises two main tasks: singular value decomposition of the input matrix $\mathbf{Z}$ followed by a shrinkage operator with level $\tau$ applying to the obtained singular values. Let $\mathcal{T}_{\tau}(x)$ denote the shrinkage or soft-thresholding operator defined as follows:

$$
\mathcal{T}_{\tau}(x)=\operatorname{sgn}(x) \max (|x|-\tau, 0)=\frac{x}{|x|} \max (|x|-\tau, 0) .
$$

For vectors or matrices, $\mathcal{T}_{\tau}(\cdot)$ is applied to each element (entrywise). The SVT operator $\mathcal{S}_{\tau}(\mathbf{Z})$ is now evaluated as

$$
\mathcal{S}_{\tau}(\mathbf{Z})=\mathbf{U} \mathcal{T}_{\tau}(\boldsymbol{\Lambda}) \mathbf{V}^{H},
$$

where $\mathbf{Z}=\mathbf{U} \boldsymbol{\Lambda} \mathbf{V}^{H}$ is the singular value decomposition of $\mathbf{Z}$. The $\ell_{1}$ regularized minimization (24) is solved by the following forward gradient and backward proximal 
steps,

$$
\begin{aligned}
\mathbf{b}_{t} & =\mathbf{s}_{t}-\beta_{t} \mathbf{D}^{H}\left(\mathbf{D} \mathbf{s}_{t}-\mathcal{A}\left(\mathbf{Z}_{t}-\mathbf{Z}_{t+1}^{w}\right)\right), \\
\mathbf{s}_{t+1} & =\arg \min _{\mathbf{s}}\left\{\frac{1}{2}\left\|\mathbf{b}_{t}-\mathbf{s}\right\|_{2}^{2}+\beta_{t} \lambda\|\mathbf{s}\|_{1}\right\},
\end{aligned}
$$

where the stepsize $\beta_{t}>0$ can be set to $\beta_{t}=1 / C$ with $\mathrm{C}=\|\mathbf{D}\|_{2}^{2}$ to ensure the convergence [27]. Problem (29) has a closed-form solution equivalent to the shrinkage operator,

$$
\mathbf{s}_{k+1}=\mathcal{T}_{\beta_{t} \lambda}\left(\mathbf{b}_{t}\right) \text {. }
$$

In summary, the PFBS-based iterative algorithm to solve Problem (15) is provided in Table I, which is referred as Algorithm 1. The input into Algorithm 1 includes the measurement vector $\mathbf{y}$, regularization parameters $\gamma, \lambda$, and a tolerance tol. Setting values for these parameters is described in Section 4. It can be observed that Algorithm 1 performs gradient computation (Step 2), followed by the proximal evaluations of SVT operator for wall clutter estimation (Step 3) and shrinkage operator for target image reconstruction (Step 4). Evaluations of these two operators are the most time-consuming steps and thereby forming the computational complexity of Algorithm 1. The computational complexity of the SVT operation in Step 3 is $\mathcal{O}\left(M N^{2}\right)$, and the time complexity of the shrinkage operator in Step 4 is $\mathcal{O}(Q)$. Thus, the overall computation complexity of each iteration is $\mathcal{O}\left(M N^{2}+Q\right)$.

It is worth noting here that in the minimization, the wall clutter matrix is estimated via SVT applied to the data matrix in which the recent estimated target component has been fixed and subtracted. Likewise, the scene image is reconstructed by applying the shrinkage operator to the measurement vector in which the recent estimated wall component is fixed and segregated. Algorithm 1 terminates after it reaches a local optimum. We implement this termination condition as the change of the cost function is very small (Step 5). After convergence, the formed target image is obtained by reshaping the column vector $\mathbf{s}$ into a $2-\mathrm{D}$ matrix.

\section{Experimental Results and Analysis}

This section presents experimental evaluation for the proposed rank-deficient and sparse approach using simulated and real radar data. The performance of the proposed approach is tested under different sensing conditions, especially when the data measurements are reduced drastically. Comparison results with other existing compressive TWRI models are also provided. First, Subsection 4.1 describes results using synthetic data. This is followed by the experimental results with real radar data in Subsection 4.2.

\subsection{Experimental Results with Synthetic Data}

4.1.1 Simulation setup: A synthetic aperture radar (SAR) system was simulated for TWR data acquisition. The transceiver was placed parallel to a $0.15 \mathrm{~m}$ thick concrete wall, at a standoff distance of $1 \mathrm{~m}$. It is moved horizontally along the wall to synthesize a linear
Table I

Algorithm 1: Wall-Clutter Removal and Target-Image Estimation in Compressive TWRI using the Proximal ForWARd-BAcKWARd SplitTing Technique

1) Initialize $\mathbf{Z}_{0}^{w} \leftarrow \mathcal{A}^{*}(\mathbf{y}), \mathbf{s}_{0} \leftarrow \mathbf{0}$, and $t \leftarrow 0$.

2) Compute gradient evaluation using (21):

$\mathbf{Z}_{t} \leftarrow \mathbf{Z}_{t}^{w}+\mathcal{A}^{*}\left(\mathbf{D} \mathbf{s}_{t}\right)-\mathcal{A}^{*}\left(\mathcal{A}\left(\mathbf{Z}_{t}^{w}\right)+\mathbf{D} \mathbf{s}_{t}-\mathbf{y}\right)$.

3) Perform wall clutter estimation using (25): $\mathbf{Z}_{t+1}^{w} \leftarrow \mathcal{S}_{\gamma}\left(\mathbf{Z}_{t}-\mathcal{A}^{*}\left(\mathbf{D} \mathbf{s}_{t}\right)\right)$.

4) Reconstruct an image of the targets using (28) and (30): $\mathbf{b}_{t} \leftarrow \mathbf{s}_{t}-\beta_{t} \mathbf{D}^{H}\left(\mathbf{D} \mathbf{s}_{t}-\mathcal{A}\left(\mathbf{Z}_{t}-\mathbf{Z}_{t+1}^{w}\right)\right)$, $\mathbf{s}_{t+1} \leftarrow \mathcal{T}_{\beta_{t} \lambda}\left(\mathbf{b}_{t}\right)$.

5) Compute the objective function $f\left(\mathbf{Z}_{t+1}^{w}, \mathbf{s}_{t+1}\right)$ using (15), if $\frac{\left|f\left(\mathbf{Z}_{t+1}^{w}, \mathbf{s}_{t+1}\right)-f\left(\mathbf{Z}_{t}^{w}, \mathbf{s}_{t}\right)\right|}{\left|f\left(\mathbf{Z}_{t}^{w}, \mathbf{s}_{t}\right)\right|}<$ tol then terminate the algorithm, otherwise increase $t \leftarrow t+1$ and go to Step 2 .

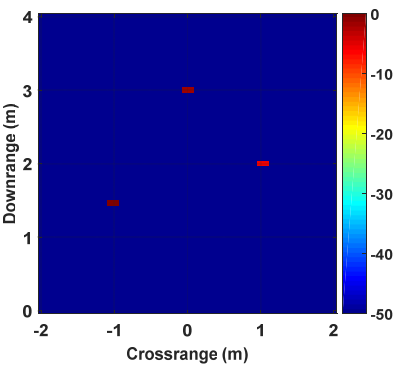

(a)

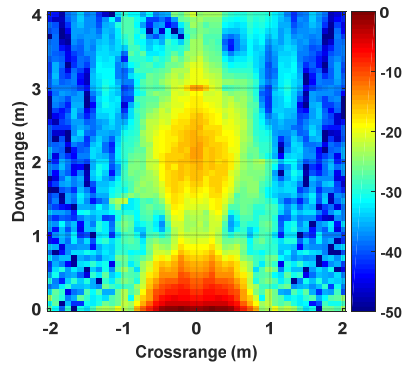

(b)
Figure 1. The behind-wall target space: (a) ground-truth target image, (b) image formed by DS beamforming technique, Equation (9), using full data volume $\mathbf{Z}$.

aperture consisting of $N=25$ elements, with a spacing between elements of $0.05 \mathrm{~m}$. A stepped-frequency signal consisting of $M=201$ frequencies, ranging from 1 to $3 \mathrm{GHz}$, with $10-\mathrm{MHz}$ frequency step, was used to scan the scene. The scene contains $(P=3)$ targets and the front wall. The three targets (each covering 2 pixels) placed behind the wall are centered at positions $p_{1}=$ $(-1 \mathrm{~m}, 1.5 \mathrm{~m}), p_{2}=(0 \mathrm{~m}, 3 \mathrm{~m}), p_{3}=(1 \mathrm{~m}, 2 \mathrm{~m})$. The downrange and crossrange of the scene extend from 0 to $4 \mathrm{~m}$, and -2 to $2 \mathrm{~m}$, respectively. The target reflection coefficients are $\sigma_{p_{1}}=1, \sigma_{p_{2}}=0.8$, and $\sigma_{p_{3}}=0.5$. The wall reflections, on the other hand, are dominant those of the targets. The wall coefficient was set to $\sigma_{w}=10$, and the number of wall reverberations is $R=48$. The pixel size was set to the Rayleigh resolution of the radar, which gives an image of size $53 \times 48$ pixels, i.e., the number of total pixels $Q=53 \times 48=2,544$.

With these settings, the wall matrix $\mathbf{Z}^{w}$ and target matrix $\mathbf{Z}^{t}$ of size $M \times N=201 \times 25$ were generated using Equations (2) and (3), respectively. Due to the column dependency, $\mathbf{Z}^{w}$ is a low-rank matrix with rank $J=1$. The received radar matrix $\mathbf{Z}$ was generated using Equation (5), in which the component $\mathbf{Y}$ was assumed to follow white Gaussian noise (WGN) with target (signal)-to-noise ratio $(\mathrm{SNR})=10 \mathrm{~dB}$. For references, Figure 1 depicts the ground-truth image and the DS 
beamforming image, Equation (9), reconstructed using the full measurement $\mathbf{Z}$. It is evident from Figure 1(b) that the wall clutter dominates the targets, making target detection impossible. Note that in this article, the target images are plotted with the maximum intensity value normalized to $0 \mathrm{~dB}$.

\subsubsection{Clutter mitigation $\mathcal{E}$ target image reconstruction:} In the first experiment, we evaluate the performance of the proposed low-rank and sparse regularized approach under incomplete data. The reduced dataset comprising only $50 \%$ of the full data volume was generated by randomly selecting half the frequencies at all antenna locations ( $K=2,525$ out of the full 5,025 samples). Thus, the input measurement vector $\mathbf{y}$ is of size $2,525 \times 1$. The resulting dictionary $\mathbf{D}$ is overcomplete with the size of $K \times Q=2,525 \times 2,544$. Using $\mathbf{y}$ and $\mathbf{D}$, clutter mitigation and target image reconstruction can be performed with Algorithm 1. This algorithm requires three parameters $\gamma, \lambda$, and tol, which need to be selected appropriately. Parameter $\gamma$ controls the importance of the low-rank term and uses in SVT to estimate the low-rank wall clutter matrix, see Step 3 in Algorithm 1. Setting $\gamma$ to a very large value, e.g., $\gamma=\left\|\mathcal{A}^{*}(\mathbf{y})\right\|_{2}$, leads to the solution of $\mathbf{Z}^{w}$ being rank 0 , whereas choosing a small value, e.g., $\gamma=10^{-4}\left\|\mathcal{A}^{*}(\mathbf{y})\right\|_{2}$ makes the algorithm converge very slowly. The values $10^{-4}\left\|\mathcal{A}^{*}(\mathbf{y})\right\|_{2}$ and $\left\|\mathcal{A}^{*}(\mathbf{y})\right\|_{2}$ can be regarded as the lower and upper bounds for $\gamma$, respectively. Here, in the experiments, $\gamma$ was set to $\gamma=10^{-2}\left\|\mathcal{A}^{*}(\mathbf{y})\right\|_{2}$. While $\gamma$ controls the rank of matrix $\mathbf{Z}^{w}$, the parameter $\lambda$ guarantees the sparsity level of the target image s. For $\lambda \geq\left\|\mathbf{D}^{H} \mathbf{y}\right\|_{\infty}$, the unique solution to Problem (15) for $\mathbf{s}$ is the zero matrix. In the following experiments, $\lambda$ was set to $\lambda=10^{-2}\left\|\mathbf{D}^{H} \mathbf{y}\right\|_{\infty}$. The algorithm converges if the relative change of the objective function is smaller than tol $=10^{-6}$ (see Step 5 in Algorithm 1).

Figure 2 shows the realization of the target image $\mathbf{s}_{t}$ estimated by Algorithm 1 at different iterations. It can be observed that after few initial iterations, the targets are undetectable due to the dominance of the strong wall returns, as illustrated in Figures 2(a) and (b). When further iterations are reached, the targets become realizable, see Figure 2(c). The targets are localized after convergence (126 iterations), as demonstrated in Figure 2(d), where target pixels are visible and wall clutter as well as background noise are significantly mitigated.

To quantify the quality of wall clutter suppression and image reconstruction, the target-to-clutter ratio (TCR) is used as a performance measure [3,11,13]. Let $A_{t}$ and $A_{c}$ be, respectively, the regions of target and clutter on the reconstructed image $I$, and let $N_{t}$ and $N_{c}$ be, respectively, the number of target and clutter pixels. The TCR (in $\mathrm{dB}$ ) measures the ratio of the average powers of the target region over the clutter region:

$$
\mathrm{TCR}=10 \log _{10}\left(\frac{\frac{1}{N_{t}} \sum_{q \in A_{t}}\left|I_{q}\right|^{2}}{\frac{1}{N_{c}} \sum_{q \in A_{c}}\left|I_{q}\right|^{2}}\right) .
$$

The target region is chosen in the vicinity of the true

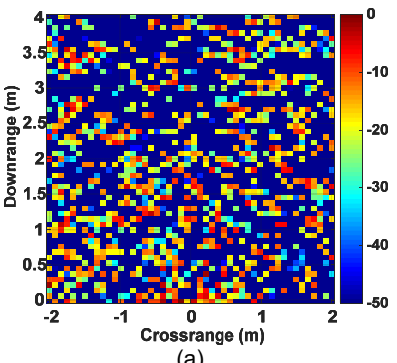

(a)

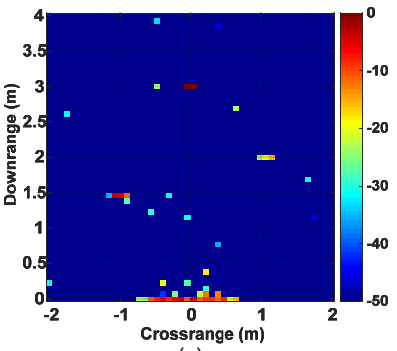

(c)

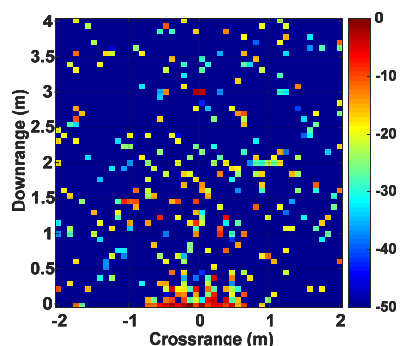

(b)

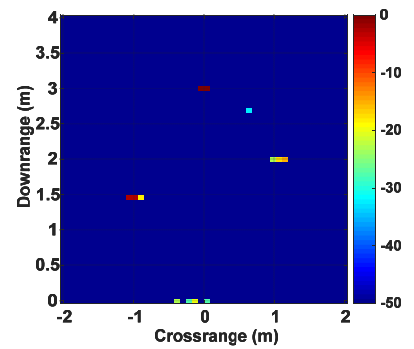

(d)
Figure 2. The realization of indoor target images reconstructed by Algorithm 1: (a) target image $\mathbf{s}_{30}$ estimated at the 30th iteration, (b) target image $\mathbf{s}_{70}$ estimated at the 70 th iteration, (c) target image $\mathbf{s}_{90}$ estimated at the 90 th iteration, and $(\mathrm{d})$ target image $\mathbf{s}_{126}$ estimated at the 126th iteration.
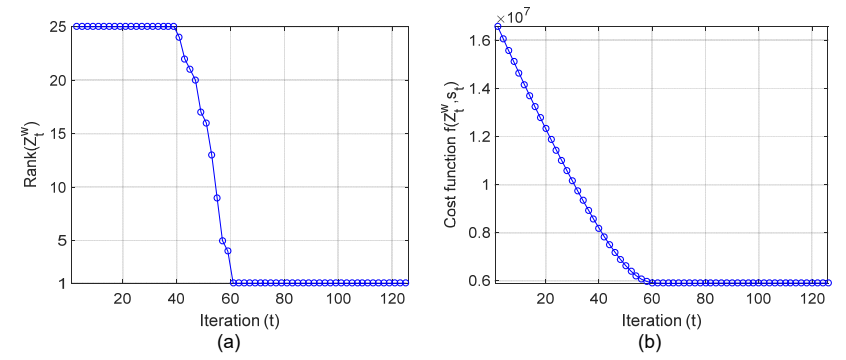

Figure 3. Evolution of the rank values of the wall component $\mathbf{Z}^{w}$ and the objective function $f\left(\mathbf{Z}^{w}, \mathbf{s}\right)$ during minimization: (a) the rank values of matrix $\mathbf{Z}^{w}$ as a function of the number of iterations, (b) $f\left(\mathbf{Z}^{w}, \mathbf{s}\right)$ as a function of the number of iterations.

targets, and the clutter region is defined as the entire image, without the target region. Using (31), we compute the TCRs for images shown in Figure 2. As expected, the TCR values increase from $-0.38 \mathrm{~dB}$ for Figure 2(a), $16.09 \mathrm{~dB}$ for Figure 2(b), to $26.69 \mathrm{~dB}$ for Figure 2(c), and $43.09 \mathrm{~dB}$ for Figure 2(d).

Further insights into the proposed optimization model can be observed from the change of the rank values for the wall component matrix $\mathbf{Z}^{w}$, shown in Figure 3(a) and the evolution of the objective function $f\left(\mathbf{Z}^{w}, \mathbf{s}\right)$, shown in Figure 3(b). As demonstrated in Figure $3(\mathrm{a})$, the rank value starts at 25 , i.e., $\min (201,25)$ and achieves a value of 1 at the steady-state. Likewise, the cost function is monotone-decreasing and converges well after 126 iterations.

4.1.3 Comparisons with different CS-based clutter mitigation $\mathcal{E}$ image reconstruction: This experiment aims to evaluate the performance of the proposed lowrank and sparse approach in comparison with other existing CS-based imaging methods. For comparison, we implement the existing direct CS and multistage CS-based approaches for wall clutter mitigation and target image reconstruction using 50\% reduced dataset 


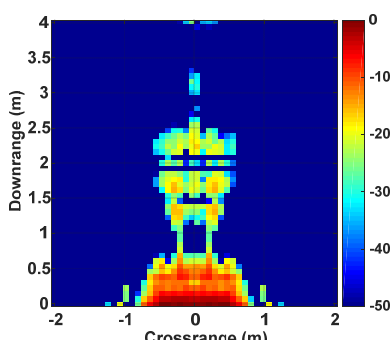

(a)

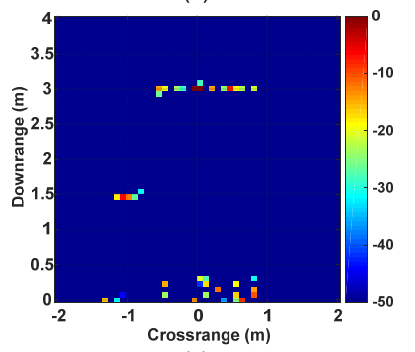

(c) (b)

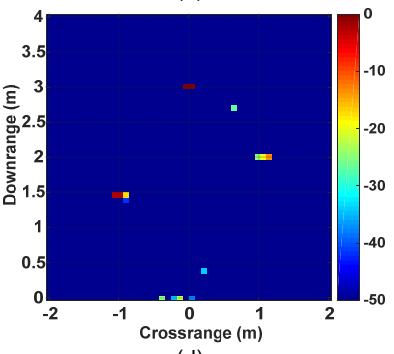

(d)

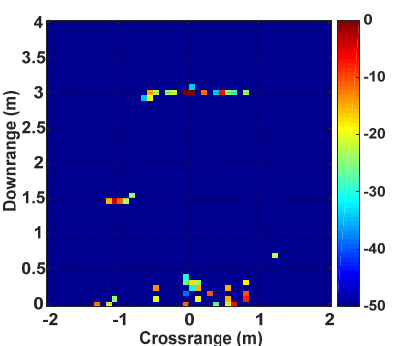

Figure 4. Images produced by different CS-based wall clutter reduction and target image reconstruction methods using $50 \%$ of full measurements: (a) direct $\ell_{1}$-norm minimization without clutter mitigation, (b) multistage CS approach with signal estimation, spatial filtering, and $\ell_{1}$-norm minimization, (c) multistage CS approach with signal estimation, subspace projection, and $\ell_{1}$-norm minimization, and (d) proposed joint nuclear-norm and $\ell_{1}$-norm method.

acquired in the same manner as in Subsection 4.1.2. While the direct CS method reconstructs a target image from the raw reduced data vector $\mathbf{y}$, the existing multistage approaches first perform data recovery, then employ a spatial filtering [15] or a subspace projection technique [16] for wall-clutter mitigation, i.e., removing the wall component $\mathbf{Z}^{w}$, and finally reconstruct a target image by solving the $\ell_{1}$-norm regularized problem in (10) using the wall-clutter subtracted data.

Figure 4 shows the target images formed by the different imaging methods. Without clutter removal, the direct CS method forms an image shown in Figure 4(a). Clearly, strong wall clutter dominates the targets, making target localization very difficult. On the other hand, by using the spatial filtering for wall clutter migration, the multistage CS approach produces the target image, shown in Figure 4(b), in which strong wall clutter has been significantly mitigated, but this image still contains a high level of false alarms. Figure 4(c) presents the image reconstructed by the multistage CS method, but the subspace projection is used, instead of the spatial filtering, for wall clutter mitigation. The quality of the target image is enhanced slightly due to better level of clutter mitigation, i.e., the subspace projection outperforms the spatial filtering in terms of clutter suppression. The proposed rank-deficient and sparse approach yields the image depicted in Figure 4(d), where the target pixels are further enhanced and clutter pixels are alleviated considerably.

The TCR values of the target images, shown in Figure 4, formed by the different CS-based methods are computed and listed in Table II. As we expect from the visual interpretation, the proposed joint nuclear-norm and $\ell_{1}$-norm approach significantly enhances the image quality in terms of TCR; it yields the target image with
Table II

Performances of Different Wall Clutter Mitigation and Target Image Formation Methods in Terms of TCR using 50\% OF Full MEASUREMENTS

\begin{tabular}{|l|c|}
\hline \hline $\begin{array}{l}\text { Clutter mitigation \& image formation } \\
\text { methods }\end{array}$ & TCR (dB) \\
\hline $\begin{array}{l}\text { Direct } \ell_{1} \text { min. without clutter } \\
\text { mitigation }[8,9]\end{array}$ & -19.22 \\
\hline $\begin{array}{l}\text { Multistage signal esti. \& spatial filt. } \\
\& \ell_{1} \text { min. [11, 15] }\end{array}$ & 29.19 \\
\hline $\begin{array}{l}\text { Multistage signal esti. \& sub. proj. } \\
\& \ell_{1} \text { min. [11, 16] }\end{array}$ & 30.60 \\
\hline $\begin{array}{l}\text { Proposed rank-deficient and sparsity } \\
\text { approach }\end{array}$ & $\mathbf{4 2 . 3 3}$ \\
\hline \hline
\end{tabular}

a TCR value of $42.33 \mathrm{~dB}$, the highest TCR value among those of the evaluated CS-based imaging approaches.

4.1.4 Performance under different noise levels: In this experiment, the performance of the proposed model is examined under different noisy sensing conditions. In doing so, we use the $50 \%$ dataset generated in the same protocol as in Subsection 4.1.2, but the complex WGN component $\mathbf{Y}$ is added to the received radar signals with SNR varying from -15 to $15 \mathrm{~dB}$. For each noise level, the proposed model is evaluated, and the TCR of the formed images, the rank, and the number of iterations are recorded for 100 trials. Table III lists the averaged computational results for all trials. It is evident that the algorithm estimates the rank of the wall matrix consistently even under highly corrupted noise. It also yields good image quality in terms of TCR for all noisy levels. However, more iterations are required for convergence under highly corrupted noise levels.

4.1.5 Effects of incomplete measurements: This experiment aims to test the effects of reducing measurements on the performance of the proposed algorithm. For this purpose, different incomplete datasets comprising 20\%, $25 \%, \ldots, 50 \%$ of the full measurements are generated by selecting measurements randomly. These reduced subsets, corrupted by noise with $\mathrm{SNR}=10 \mathrm{~dB}$, are used as input for evaluating the proposed model. For each dataset, the rank of the wall component $\mathbf{Z}^{w}$, the value of the cost function, the relative change of the objective function, the number of iterations, and TCR values of the formed images are recorded after convergence. Table IV presents the results for all datasets, averaged over 50 trials. Three noticeable observations made from the results include: (1) the proposed algorithm converges and estimates the rank correctly for the all levels of reduced datasets, (2) far fewer iterations are needed for convergence when more data samples are available, and (3) the TCR values enhance together with the use of the number of measurements.

\subsection{Experimental Results with Real Data}

4.2.1 Experimental setup: Real radar datasets were collected from a scene, shown in Figure 5, that consists of one dihedral target placed behind a wooden wall. This behind-wall scene is sensed by a 81-element linear 
Table III

Computational Results of the Proposed Low-rank and Sparse Regularized Model for Different Noise Levels using 50\% Of the Total Measurements

\begin{tabular}{|c|c|c|c|c|}
\hline \hline SNR (dB) & \multirow{2}{*}{$\operatorname{Rank}\left(\mathbf{Z}^{w}\right)$} & $\frac{\left|f\left(\mathbf{Z}_{t+1}^{w}, \mathbf{s}_{t+1}\right)-f\left(\mathbf{Z}_{t}^{w}, \mathbf{s}_{t}\right)\right|}{\left|f\left(\mathbf{Z}_{t}^{w}, \mathbf{s}_{t}\right)\right|}$ & Iterations & TCR (dB) \\
\hline-15 & 1 & $9.63 \times 10^{-7}$ & 155 & 19.45 \\
\hline-10 & 1 & $9.31 \times 10^{-7}$ & 143 & 30.96 \\
\hline-5 & 1 & $8.86 \times 10^{-7}$ & 135 & 40.60 \\
\hline 0 & 1 & $8.54 \times 10^{-7}$ & 134 & 43.49 \\
\hline 5 & 1 & $8.34 \times 10^{-7}$ & 134 & 43.58 \\
\hline 10 & 1 & $8.56 \times 10^{-7}$ & 132 & 44.75 \\
\hline 15 & 1 & $8.31 \times 10^{-7}$ & 130 & 45.86 \\
\hline
\end{tabular}

Table IV

Computational Results of the Low-rank and Sparse Model for Different Reduced Datasets

\begin{tabular}{|c|c|c|c|c|}
\hline \hline \multirow{2}{*}{ Dataset } & \multirow{2}{*}{$\boldsymbol{\operatorname { a n k }}\left(\mathbf{Z}^{w}\right)$} & $\frac{\left|f\left(\mathbf{Z}_{t+1}^{w}, \mathbf{s}_{t+1}\right)-f\left(\mathbf{Z}_{t}^{w}, \mathbf{s}_{t}\right)\right|}{\left|f\left(\mathbf{Z}_{t}^{w}, \mathbf{s}_{t}\right)\right|}$ & Iterations & TCR (dB) \\
\hline $20 \%$ & 1 & $6.90 \times 10^{-7}$ & 567 & 25.07 \\
\hline $25 \%$ & 1 & $7.97 \times 10^{-7}$ & 368 & 32.26 \\
\hline $30 \%$ & 1 & $7.59 \times 10^{-7}$ & 253 & 34.07 \\
\hline $35 \%$ & 1 & $8.01 \times 10^{-7}$ & 203 & 37.64 \\
\hline $40 \%$ & 1 & $7.71 \times 10^{-7}$ & 168 & 38.87 \\
\hline $45 \%$ & 1 & $8.05 \times 10^{-7}$ & 144 & 42.59 \\
\hline $50 \%$ & 1 & $7.84 \times 10^{-7}$ & 134 & 44.79 \\
\hline \hline
\end{tabular}

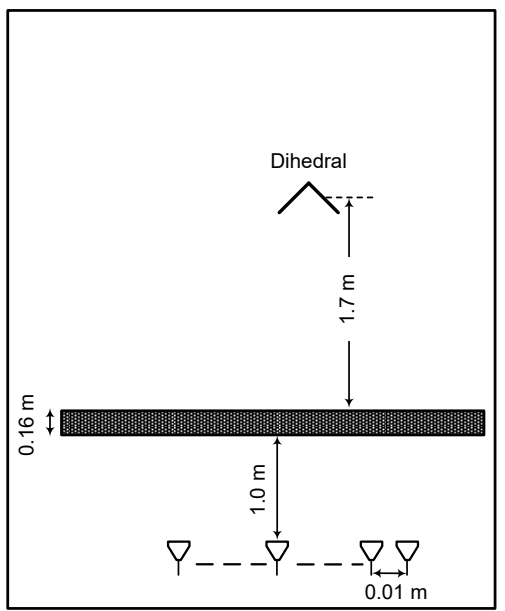

Figure 5. Layout of the imaged scene containing a wooden wall and one dihedral used as target for through-the-wall radar data acquisition.

antenna array synthesized by a monostatic radar system with the inter-element spacing of $0.01 \mathrm{~m}$. The radar system transceives a 801-step frequency signal covering the bandwidth of [1-3 GHz]. This stepped-frequency SAR system was positioned in front of the $0.16 \mathrm{~m}$-thick wooden wall, at a standoff distance of $1.0 \mathrm{~m}$.

4.2.2 Clutter mitigation $\mathcal{E}$ image reconstruction: This experiment evaluates the proposed rank-deficient and sparse approach under generic CS operations where both antennas and frequencies are highly compressed. CS radar imaging acquires a reduced dataset, instead of full data volume, by randomly selecting only half of the available antennas (41 out of 81). Each selected antenna uses only $20 \%$ (160 out of 801$)$ frequencies, which are randomly sampled. Thus, the used dataset constitutes only $10 \%$ of the full data volume. For comparison, we also implement the existing direct CS and multistage CS-based approaches for wall clutter mitigation and target image reconstruction using the same reduced dataset.

Figure 6 depicts the target images reconstructed using the different imaging methods. The direct CS method yields a degraded image shown in Figure 6(a) due to the presence of strong wall interferences, hindering target identification. The multistage CS-based methods with the spatial filtering and subspace projection produce the target images in Figures 6(b) and (c), respectively. In these formed target images, strong wall clutter is suppressed, which enables the target localization. The proposed joint low-rank and sparse model gives the image shown in Figure 6(d), where the target pixels are highlighted and background clutter are substantially reduced.

Table V presents the performance measure of TCRs by the different CS-based techniques. It can be observed from the table that the proposed joint nuclear-norm and $\ell_{1}$-norm approach outperforms the exisiting CS-based imaging techniques in terms of TCR; it has the highest TCR value of $29.97 \mathrm{~dB}$, followed by the multistage CS with subspace projection of $16.74 \mathrm{~dB}$, multistage CS with spatial filtering of $6.05 \mathrm{~dB}$, and direct CS of $1.13 \mathrm{~dB}$. 


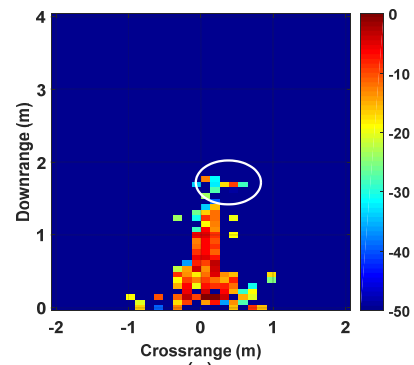

(a)

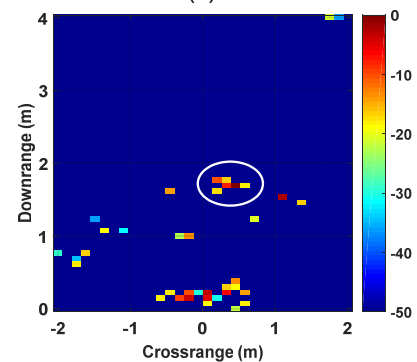

(c)

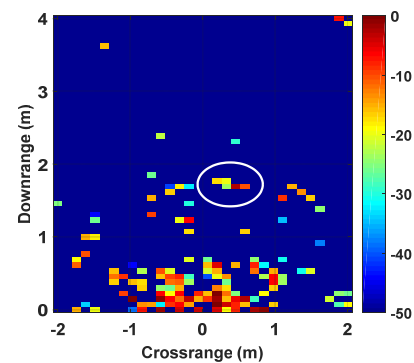

(b)

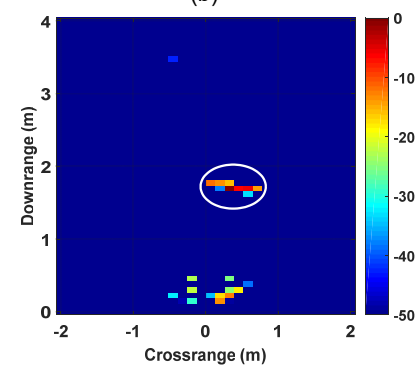

(d)

Figure 6. Images produced by different CS-based wall clutter reduction and target image reconstruction methods with 50\% antennas and $20 \%$ frequencies (collectively represent only $10 \%$ of full measurements): (a) direct $\ell_{1}$-norm minimization without clutter mitigation, (b) multistage CS approach with signal estimation, spatial filtering, and $\ell_{1}$-norm minimization, (c) multistage CS approach with signal estimation, subspace projection, and $\ell_{1}$-norm minimization, and $(\mathrm{d})$ proposed joint nuclear-norm and $\ell_{1}$-norm method.

Table V

Target-to-Clutter Ratio Performances for the Images Produced by Several CS-based Techniques with 50\% Antennas AND 20\% FREQUENCIES (COLleCtively REPRESENT ONLY 10\% OF FULL MEASUREMENTS)

\begin{tabular}{|l|c|}
\hline \hline $\begin{array}{l}\text { Clutter mitigation \& image formation } \\
\text { methods }\end{array}$ & TCR (dB) \\
\hline $\begin{array}{l}\text { Direct } \ell_{1} \text { min. without clutter } \\
\text { mitigation }[8,9]\end{array}$ & 1.13 \\
\hline $\begin{array}{l}\text { Multistage signal esti. \& spatial filt. } \\
\& \ell_{1} \text { min. [11, 15] }\end{array}$ & 6.05 \\
\hline $\begin{array}{l}\text { Multistage signal esti. \& sub. proj. } \\
\& \ell_{1} \text { min. [11, 16] }\end{array}$ & 16.74 \\
\hline $\begin{array}{l}\text { Proposed rank-deficient and sparsity } \\
\text { approach }\end{array}$ & 29.97 \\
\hline
\end{tabular}

To evaluate the capability of target detection by different wall clutter mitigation and image reconstruction methods, we apply a thresholding technique to the target images formed by the different methods shown in Figure 6. Here, the input image is partitioned into two classes: object and background. The threshold used for classification is found by maximizing the betweenclass variance. Figure 7 presents the detection results after applying the same threshold value to the form images. As expected, without wall clutter mitigation, the direct CS fails to localize the target as demonstrated in Figure 7(a). By contrast, it can be observed from Figures 7(b) and (c) that the multistage CS approach can detect the target with a high level of false alarms. The detection result in Figure 7(d) shows that the proposed model is able to localize the target well, with no appearance of false alarms.

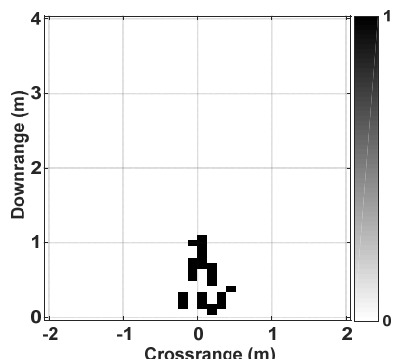

(a)

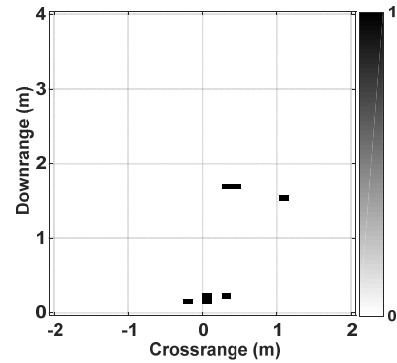

(c)

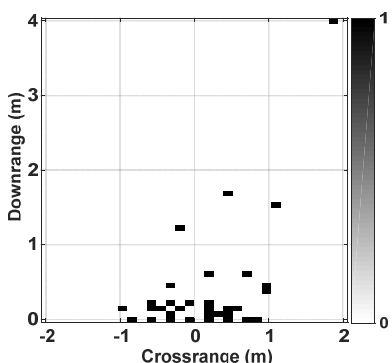

(b)

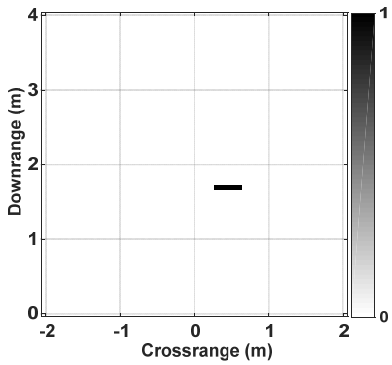

(d)
Figure 7. Detection of target by thresholding the images produced by different CS-based wall clutter reduction and target image reconstruction methods with 50\% antennas and $20 \%$ frequencies (collectively represent only $10 \%$ of full measurements): (a) direct $\ell_{1}$-norm minimization without clutter mitigation, (b) multistage CS approach with signal estimation, spatial filtering, and $\ell_{1}$-norm minimization, (c) multistage CS approach with signal estimation, subspace projection, and $\ell_{1}$-norm minimization, and (d) proposed joint nuclear-norm and $\ell_{1}$-norm method.

\section{Conclusion}

This paper introduced a rank-deficient and sparsity regularized optimization model to address two important problems of wall clutter mitigation and target image formation in compressive indoor radar imaging. The task of wall clutter suppression and target image reconstruction is formulated as a composite nuclear and $\ell_{1}$-penalized minimization problem and an iterative algorithm based on the proximal forward-backward splitting technique is developed, which captures wall clutter and yields an indoor target image simultaneously. Through extensive experimental evaluations on simulated and real radar data, we found that exploiting both rank-deficient and sparsity structures improves the accuracy of clutter suppression and enhances target detection even with highly compressed measurements. Furthermore, the proposed model enables the processing of wall and target signals jointly, which results in a significant better performance than those of multistage approaches where the wall clutter removal and target image reconstruction are performed separately.

\section{ACKNOWLEDGMENTS}

The authors would like to thank the Editor-in-Chief, Associate Editor, and three anonymous reviewers for the constructive and detailed comments that helped us improve the paper.

This research is funded by Vietnam National Foundation for Science and Technology Development (NAFOSTED) under grant number 102.01-2017.307. 


\section{REFERENCES}

[1] M. G. Amin, Ed., Through-the-Wall Radar Imaging. CRC press, 2010.

[2] F. Ahmad, M. G. Amin, and S. A. Kassam, "Synthetic aperture beamformer for imaging through a dielectric wall," IEEE Transactions on Aerospace and Electronic Systems, vol. 41, no. 1, pp. 271-283, 2005.

[3] V. H. Tang, S. L. Phung, F. H. C. Tivive, and A. Bouzerdoum, "A sparse Bayesian learning approach for through-wall radar imaging of stationary targets," IEEE Transactions on Aerospace and Electronic Systems, vol. 53, no. 5, pp. 2485-2501, 2017.

[4] M. G. Amin and F. Ahmad, "Wideband synthetic aperture beamforming for through-the-wall imaging," IEEE Signal Processing Magazine, vol. 25, no. 4, pp. 110-113, 2008.

[5] D. L. Donoho, "Compressed sensing," IEEE Transactions on Information Theory, vol. 52, no. 4, pp. 1289-1306, 2006.

[6] E. J. Candes, J. K. Romberg, and T. Tao, "Stable signal recovery from incomplete and inaccurate measurements," Communications on Pure and Applied Mathematics: A Journal Issued by the Courant Institute of Mathematical Sciences, vol. 59, no. 8, pp. 1207-1223, 2006.

[7] E. J. Candès, J. Romberg, and T. Tao, “Robust uncertainty principles: Exact signal reconstruction from highly incomplete frequency information," IEEE Transactions on Information Theory, vol. 52, no. 2, pp. 489-509, 2006.

[8] Y.-S. Yoon and M. G. Amin, "Compressed sensing technique for high-resolution radar imaging," in Proceedings of the Signal Processing, Sensor Fusion, and Target Recognition XVII, vol. 6968. International Society for Optics and Photonics, 2008, pp. 69681A.1-69681A.10.

[9] Q. Huang, L. Qu, B. Wu, and G. Fang, "UWB throughwall imaging based on compressive sensing," IEEE Transactions on Geoscience and Remote Sensing, vol. 48, no. 3, pp. 1408-1415, 2009.

[10] V. H. Tang, A. Bouzerdoum, and S. L. Phung, "Two-stage through-the-wall radar image formation using compressive sensing," Journal of Electronic Imaging, vol. 22, no. 2, pp. 021 006.1-021 006.10, 2013.

[11] E. Lagunas, M. G. Amin, F. Ahmad, and M. Nájar, "Joint wall mitigation and compressive sensing for indoor image reconstruction," IEEE Transactions on Geoscience and Remote Sensing, vol. 51, no. 2, pp. 891-906, 2012.

[12] V. H. Tang, A. Bouzerdoum, S. L. Phung, and F. H. C. Tivive, "Enhanced wall clutter mitigation for compressed through-the-wall radar imaging using joint Bayesian sparse signal recovery," in Proceedings of the IEEE International Conference on Acoustics, Speech and Signal Processing (ICASSP). IEEE, 2014, pp. 7804-7808.

[13] F. Ahmad, J. Qian, and M. G. Amin, "Wall clutter mitigation using discrete prolate spheroidal sequences for sparse reconstruction of indoor stationary scenes," IEEE Transactions on Geoscience and Remote Sensing, vol. 53, no. 3, pp. 1549-1557, 2014.

[14] A. Bouzerdoum, F. H. C. Tivive, and V. H. Tang, "Multipolarization through-the-wall radar imaging using joint Bayesian compressed sensing," in Proceedings of the 19th International Conference on Digital Signal Processing. IEEE, 2014, pp. 783-788.

[15] Y.-S. Yoon and M. G. Amin, "Spatial filtering for wallclutter mitigation in through-the-wall radar imaging," IEEE Transactions on Geoscience and Remote Sensing, vol. 47, no. 9, pp. 3192-3208, 2009.

[16] F. H. C. Tivive, A. Bouzerdoum, and M. G. Amin, “A subspace projection approach for wall clutter mitigation in through-the-wall radar imaging," IEEE Transactions on Geoscience and Remote Sensing, vol. 53, no. 4, pp. 21082122, 2014.

[17] H. Van Tang and V.-G. Nguyen, "A rank-deficient and sparse penalized optimization model for compressive indoor radar imaging," in Proceedings of the 3rd Interna- tional Conference on Recent Advances in Signal Processing Telecommunications \& Computing (SigTelCom). IEEE, 2019, pp. $48-52$

[18] P. L. Combettes and V. R. Wajs, "Signal recovery by proximal forward-backward splitting," SIAM J. Multiscale Multiscale Modeling \& Simulation, vol. 4, no. 4, pp. 1168-1200, 2005.

[19] P. L. Combettes and J.-C. Pesquet, "Proximal splitting methods in signal processing," in Fixed-point algorithms for inverse problems in science and engineering. Springer, 2011, pp. 185-212.

[20] N. Parikh and S. Boyd, "Proximal algorithms," Foundations and Trends ${ }^{\circledR}$ in Optimization, vol. 1, no. 3, pp. 123231, 2013.

[21] V. H. Tang, A. Bouzerdoum, S. L. Phung, and F. H. C. Tivive, "Enhanced through-the-wall radar imaging using Bayesian compressive sensing," in Proceedings of the SPIE Compressive Sensing II, vol. 8717. International Society for Optics and Photonics, 2013, pp. 87170I.1-87 170I.12.

[22] V. H. Tang, A. Bouzerdoum, and S. L. Phung, "Multipolarization through-wall radar imaging using low-rank and jointly-sparse representations," IEEE Transactions on Image Processing, vol. 27, no. 4, pp. 1763-1776, 2017.

[23] S. Ma, D. Goldfarb, and L. Chen, "Fixed point and Bregman iterative methods for matrix rank minimization," Mathematical Programming, vol. 128, no. 1-2, pp. 321-353, 2011.

[24] J.-F. Cai, E. J. Candès, and Z. Shen, "A singular value thresholding algorithm for matrix completion," SIAM Journal on Optimization, vol. 20, no. 4, pp. 1956-1982, 2010.

[25] R. T. Rockafellar, Convex analysis. Princeton University Press, 1970, no. 28.

[26] K. Lee and Y. Bresler, "ADMiRA: Atomic decomposition for minimum rank approximation," IEEE Transactions on Information Theory, vol. 56, no. 9, pp. 4402-4416, 2010.

[27] A. Beck and M. Teboulle, "A fast iterative shrinkagethresholding algorithm for linear inverse problems," SIAM Journal on Imaging Sciences, vol. 2, no. 1, pp. 183202, 2009.

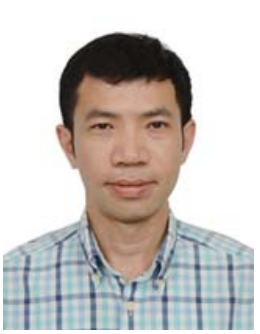

Van Ha Tang received the B.Eng. degree in 2005 and M.Eng. degree in 2008, all in computer engineering, from Le Quy Don Technical University, Hanoi, Vietnam. In 2016, he obtained the Ph.D. degree in computer engineering from the University of Wollongong, Australia. Since 2016, he has been working as a Postdoctoral Research Fellow in the School of Electrical Computer and Telecommunications Engineering, University of Wollongong. Dr. Tang is now with the Faculty of Information Technology, Le Quy Don Technical University, Hanoi, Vietnam. His research interests include radar imaging and through-wall radar signal processing, statistical and probabilistic modeling, and inverse problems in image and signal processing.

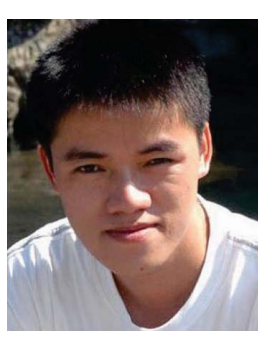

Van-Giang Nguyen received his B.S. degree in computer science from Le Quy Don Technical University, Hanoi, Vietnam, in 2005, and his M.Sc. and Ph.D. degrees in electronic engineering from Paichai University, Daejeon, Korea, in 2009 and 2012, respectively. Since 2013, he has been with the Department of Information Systems, Le Quy Don Technical University, Hanoi, Vietnam. His current research interests include image processing, computer vision, machine learning and their applications to medical physics and medical imaging. 